\title{
Analysis of Tomato Leaf Disease Identification Techniques
}

\author{
Gaurav Chopra ${ }^{1 *}$, Pawan Whig ${ }^{2}$ \\ ${ }^{1}$ Research Scholar, The Research world, India \\ ${ }^{2}$ Vivekananda Institute of Professional Studies, New Delhi-110034, India \\ ${ }^{1}$ gauravchopracg@gmail.com *; ${ }^{2}$ pawanwhig@ gmail.com; \\ * corresponding author
}

\section{A R T I CLE I N F O}

Article History

Received March 02, 2021

Revised April 08, 2021

Accepted June 20, 2021

Available Online August 14, 2021

\section{Keywords:}

Plant Disease

Neural Networks;

Tomato Leaf Disease

\section{Correspondence:}

Telephon: +919811908699

E-mail:

gauravchopracg@gmail.com

\begin{tabular}{l} 
ABSTRACT \\
\hline India loses thousands of metric tons of tomato crop every year due to pests and \\
diseases. Tomato leaf disease is a major issue that causes significant losses to \\
farmers and possess a threat to the agriculture sector. Understanding how does an \\
algorithm learn to classify different types of tomato leaf disease will help scientist \\
and engineers built accurate models for tomato leaf disease detection. \\
Convolutional neural networks with backpropagation algorithms have achieved \\
great success in diagnosing various plant diseases. However, human benchmarks \\
in diagnosing plant disease have still not been displayed by any computer vision \\
method. Under different conditions, the accuracy of the plant identification system \\
is much lower than expected by algorithms. This study performs analysis on \\
features learned by the backpropagation algorithm and studies the state-of-the-art \\
results achieved by image-based classification methods. The analysis is shown \\
through gradient-based visualization methods. In our analysis, the most \\
descriptive approach to generated attention maps is Grad-CAM. Moreover, it is \\
also shown that using a different learning algorithm than backpropagation is also \\
possible to achieve comparable accuracy to that of deep learning models. Hence, \\
state-of-the-art results might show that Convolutional Neural Network achieves \\
human comparable accuracy in tomato leaf disease classification through \\
supervised learning. But, both genetic algorithms and semi-supervised models \\
hold the potential to built precise systems for tomato leaf detection. \\
\hline
\end{tabular}

\section{Introduction}

In India, tomato is the major horticulture crop with an estimated production of 18735.91 thousand metric tons. Tomato plant has around 7500 variants and is vulnerable to 200 pests and diseases. Other than that tomato is consumed in diverse ways, raw or cooked making it more harmful for the people who consume it. Tomato leaf disease is a major issue that causes significant losses to farmers and possesses a threat to the agriculture sector [1].

Leaf Disease Identification has been a key problem in the field of agriculture from a long time. Prediction of plant disease accurately depends upon three factors - host, environment, and pathogens [2]. Plant Disease Detection systems can be designed efficiently if there is understanding of their capabilities and the methods powering current disease identification systems. However, taking a look at research in Plant Pathology, It is shown that Plant disease identification is currently progressing at the speed of light. Startups have started developing Smartphone applications [3,4] that use imagebased algorithms to diagnose plant disease. Moreover, they also provide information on how to take care of plants efficiently. But such algorithms still lack the power to analyze plants in different lighting conditions. When there is change in an environment like cloudy, overcast, and sunny conditions algorithms efficiency reduces dramatically and it questions the precision power of algorithms have been built so far [5].

Even after providing data augmentation and more diverse training data, supervision learning is still yet to be improved. Computer vision techniques like image classification, segmentation and object detection show the potential to build a highly précised and accurate system that can identify any type 
of species of crop but it's a long way down. After the advances in deep learning various papers came that showed how to train a deep convolutional network to identify different crop species. Few years ago a paper came out in which researchers showed by training a deep convolutional neural network how it is possible to identify 14 crop species and 26 diseases with an accuracy of $99.35 \%$ on the training set and $31.4 \%$ on the test set [6]. It was a competition dataset cleaned in the lab but in real life, when farmers will be dealing with this type of system change in environment conditions will provide a speed breaker in growing healthy crops. Since then various papers adopted the method of using deep convolutional networks to identify disease on different crop species whether only using a single crop like tomato or taking thousands of crops and their species to produce state of the art results on plant disease identification [7].

To understand how back propagation algorithms train a neural network in this paper, a comprehensive analysis on gradient based visualization methods are performed that has learned to detect particular lesions which belong to a leaf disease, gradient-based visualization methods are used. The gradient based visualization methods are performed to understand which activations of neurons fired inside a trained convolutional neural network when input image is detected.

To understand how back propagation algorithms train a neural network in this paper, a comprehensive analysis on gradient based visualization methods are performed. Gradient based visualization methods help us study the process of learning of artificial neural networks. Using the gradient based methods, an input image is passed through the network and updates in gradients values are recorded. These updates are used to calculate activation values change. When we plot those activations on the image, it will show how neural network learns to detect specific type of lesion present in an image. With the help of them it is shown how backpropagation algorithm trained neurons to distinguish between different types of leaf disease. Using gradient based visualization methods to compare various activation maps of learned features and then exploring the possibility of using a different learning approach is discussed.

In the field of Explainable Computer Vision, several techniques have been proposed to study and analyze what convolutional neural networks have learned. They mostly lie in four categories of visualization methods (I) hidden layer output visualization [6], (II) feature visualization [8,9], (III) semantic dictionary [9], (IV) attention map [10]. Each of these visualization maps generates activation of layers inside a neural network.

Hidden layer output visualization methods are the simplest ones to visualize a deep neural network. In this method, an image is passed tothe $\mathrm{CNN}$ and calculation is halted at the layer of interest utilizing the same technique for each layer to extract the learned feature in interpretable form [6]. Feature Visualization [8] methods are used to visualize the feature of the CNN by observing the activation of respective neurons with a gradient ascent based approach. In this method, a random-noise image is passed to the neural network and calculation of gradient of the input image is performed. By adding gradients to the input image, the mean output of values of the neuron of interest is calculated.

Semantic Dictionary [9] is a combination of feature visualization and intermediate output visualization and Attention maps allow us to obtain spatial information about the input image. While semantic dictionaries enable better understanding of the process of image classification, Activation maps [10] allows us to comprehend how gradient updates weights of a deep neural network. Gradient based visualization methods are used to perform feature visualization and are used to extract activation maps from the network.

\section{Method}

This study focused on the visualization of the feature detecting during the process of backpropagation by a deep neural network. To do that it was divided into three sections: data preprocessing, model training and gradient based visualization. 
Data is borrowed from Plant Village Dataset; it is released under Creative Commons AttributionShareAlike 3.0 Unported (CC BY-SA 3.0). It consist of image of 38 types of crops pairs which were cleaned in lab. The images in database were taken at Experimental Research Station associated with Land Grant Universities in USA (Penn State, Florida State, Cornell and others). All the images were taken with grey or black background by placing the leaf in a piece of paper by technicians with digital camera.

In Data preprocessing stage, tomato leaf images were taken from dataset and they were resized into 224 x 224 pixels, after that each image is normalized with mean 0 and standard deviation 1 . The selections of images are done from three categories of tomato leaf disease: septoria leaf spot, bacterial spot and mosaic virus; each of this disease is caused by a different type of pathogen.

Neural Network layers were trained using Transfer Learning technique, in which last few layers of network are fine-tuned to classify different type of tomato leaf disease. With the help of transfer learning, Convolutional layers has been trained and One Cycle learning policy [11] is used for faster training of network it is done by using Fast.ai library [12]. Images were normalized with pixel values range $[0.0,0.1]$. ADAM optimization algorithm is used with categorical cross-entropy loss metric to train the neural network. After that, Gradient Based Visualization is performed to analyze the learned feature and plot the results; the following methods are adopted to analyze learned features:

1. Vanilla back-propagation [13]

2. Integrated gradients [14]

3. Guided back-propagation [15]

4. Grad-CAM [16]

The images used in this study are borrowed from Plant Village Dataset. The tomato leaf images infected by different types of pathogens are adopted from it. It consists of 10 classes: 9 types of tomato leaf diseases and healthy leaf images. With the help of such images, a convolutional neural network has been fine-tuned, it receives a three-channel input image of size 224x224 and outputs a 10-dimensional vector comprises of tomato leaf identification probability.

The selections of images are done from three categories of tomato leaf disease: septoria leaf spot, bacterial spot and mosaic virus; each of this disease is caused by a different type of pathogen. After that, comparisons between all three categories of disease are done by human and their representative maps are generated to obtain spatial information about visual interpretable hotspots. There is no metric has been devised yet to evaluate how accurate computer generated visualized maps are with human knowledge. Therefore, even if Gradient-based methods provide meaningful result to understand the process of training a network learn to classify tomato disease. It is barriers in using neural networks that human still require evaluating computer generated visualization results with professional knowledge.

With the help of transfer learning, Convolutional Neural Network has been trained and One Cycle learning policy [11] is used for faster training of network it is done by using Fast.ai library [12]. Images were normalized with pixel values range [0.0, 0.1]. ADAM optimization algorithm is used with categorical cross-entropy loss metric to train the neural network. After that, Gradient Based Visualization is performed to analyze the learned feature and plot the results.

Scripts required to reproduce the results in this study is available at the following GitHub repository: https://github.com/gauravchopracg/analysis-of-tomato-leaf-disease-identification-techniques

\section{Results and Discussion}

After training of neural network, output of each visualization method is saved and is shown below. Using the gradient based methods, an input image is passed through the network and updates in gradients values are calculated. To compare and comprehend how backpropagation updates the specific layers of the network for leaf disease detection the images are compared in table 1. Different 
classes of diseases show different activation fired when input image is passed through the network. These methods give different activation maps us to identify types of plant disease that network learned to distinguish. By using these methods we can consider spatial information and comprehend which part of the image is infected by a specific type of pathogen. Table 1 summarizes the visualization created by such methods.

Table 1. Gradients Based Visualization

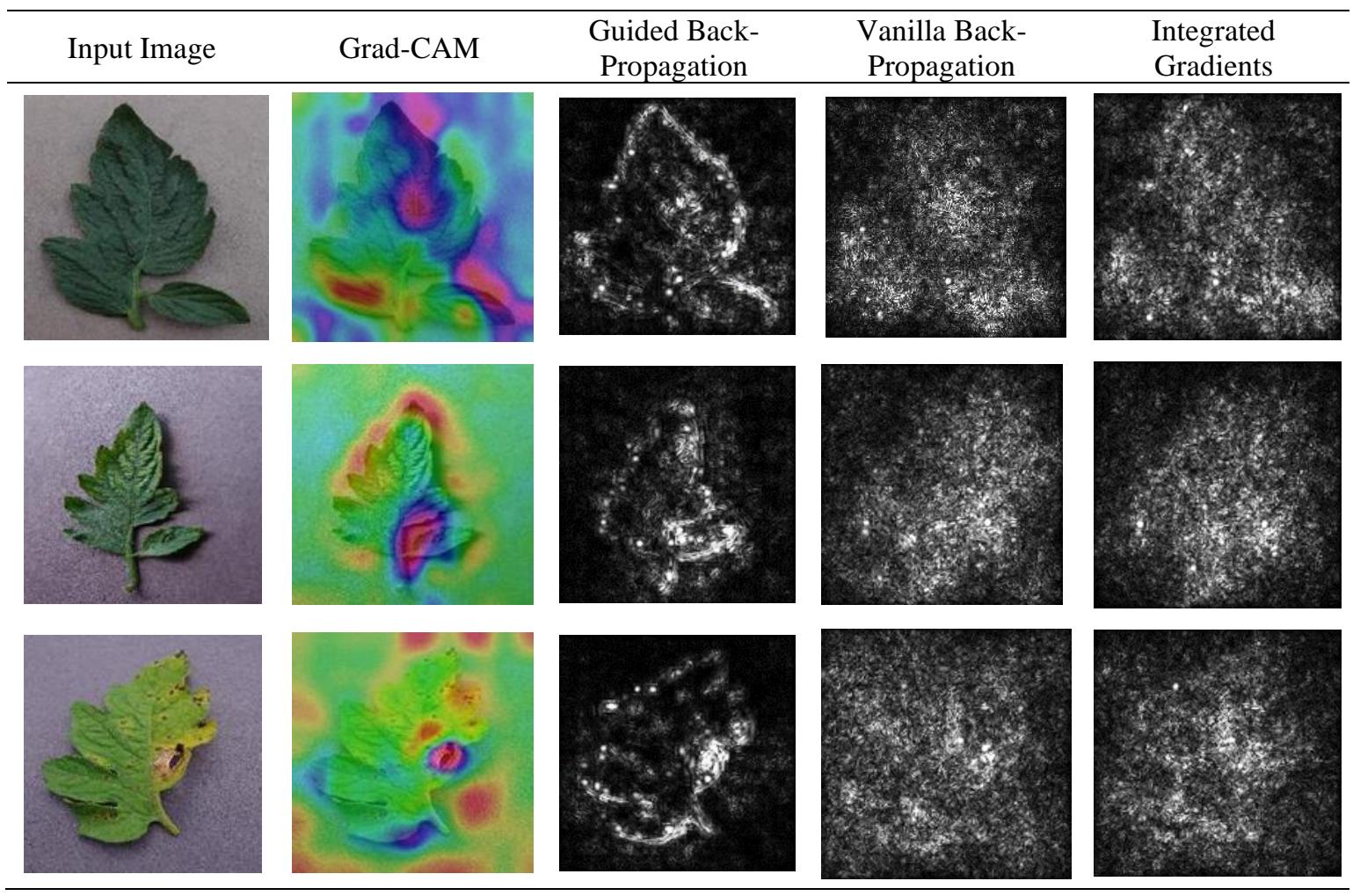

Using the visualization methods the feature learned by the neural network layers are plotted and compared. Through guided back-propagation the sensitivity of gradients has been compared [15] with vanilla back-propagation [13] and integrated gradients [14] by taking a look at table 1; both vanillaback-propagation and integrated gradients shows activation from backgrounds fired instead of neurons that are closer to lesions in the image. Both of these methods lack specificity. By using guided back-propagation alone it has been shown that a closer look at particular disease detection and part of leaf activation the network has learned to detect.

Using Grad-CAM [16] a particular activation of class has been activated when an input image belonging to it is passed through the network however, it also lacked specificity. Grad-CAM depends upon the size of intermediate output there by when generation of activation occurs through shallow layers it highlights lesions than deeper ones.

This study compares different gradient based visualization maps of infected tomato leaf diseases by studying the activation maps generated through them. Deep learning provides state of the art results in plant disease identification but traditional computer vision techniques also achieve considerable accuracy when using Tree-Based Pipeline Optimization Tool (TPOT) [17] it is possible to achieve same accuracy as done through neural networks, Tree-Based Pipeline uses genetic programming to perform classification by using plant leaf dataset with considerable amount of preprocessing, it is possible to achieve same result as given bydeep neural networks. Instead of using Supervised Learning, it is possible to use Semi Supervised Learning to perform disease identification. Since the real world data is much more complex and data scientists are not always able to provide cleaned and labeled dataset, it should be the focus to build unsupervised learning algorithms. But as it cannot be 
possible to use unsupervised learning on a dataset to reach the goal yet; there should be much more potential lies in improving biological form of learning. Last year, a new paper came out that discusses how traditional form of back propagation is biologically implausible [18]. This paper discusses a form of semi supervised learning algorithm that utilizes global inhibition in hidden layers by learning early feature detectors by using a form of biological unsupervised learning. Instead of using supervised learning with backpropagation, it implements an unusual learning rule, which is based on Hebb's idea that change of the synapse strength should be local - i.e., synapse strength should only depend on the activities of the pre and post synaptic neurons. Both of these methods provide potential in tomato leaf disease classification, and gives comparable accuracy as deep neural networks.

\section{Conclusion}

In this study, a comprehensive analysis on visualized layers of features learned through backpropagation algorithm is done. The experimental results show layers visualized through gradient based visualization methods such as Grad-CAM, guided backpropagation, vanilla backpropagation and integrated gradients. To understand what part of the input image shows activation of neurons inside the network, attention maps has been generated and detection of specific lesions is represented through them.In practice, visualization of each layer provide interpretation on learning of neural network. But using gradient based methods, this study analysis the process of backpropagation by visualizing the weights updates. The gradient descent optimization process trains a neural network, update activations by iterations and model a network to mimic human brain. Therefore, when an image is passed through the network of infected plants of different types of pathogen and their representative maps are obtained. These representative maps show us how their layers learn to classify different types of tomato disease. In this paper, the most descriptive approach to generated attention maps is Grad-CAM. It is also the simplest and cost-effective method to visualize layers.

\section{References}

[1] Strange RN, Scott PR. Plant disease: a threat to global food security. Annu Rev Phytopathol. 2005;43:83116. doi: 10.1146/annurev.phyto.43.113004.133839. PMID: 16078878.

[2] Hadwiger, Lee. Plant science review: Multiple effects of chitosan on plant systems: Solid science or hype. Plant science : an international journal of experimental plant biology. 2013;208C. 42-49. 10.1016/j.plantsci.2013.03.007.

[3] S.J. Pethybridge, S.C. Nelson. Leaf Doctor: a new portable application for quantifying plant disease severity Plant Dis., 99 (2015), pp. 1310-1316. https://doi.org/10.1094/PDIS-03-15-0319-RE.

[4] L. Hallau, M. Neumann, B. Klatt, B. Kleinhenz, T. Klein, C. Kuhn, M. Röhrig, C. Bauckhage, K. Kersting, A.K. Mahlein, U. Steiner, E.C. Oerke, Plant Pathol. 67 (2018).

[5] Esmael Hamuda, Martin Glavin, and Edward Jones. A survey of image processing techniques for plant extraction and segmentation in the field. 2016. Comput. Electron. Agric. 125, C (July 2016), 184-199. DOI:https://doi.org/10.1016/j.compag.2016.04.024.

[6] Mohanty SP, Hughes DP, Salathé M. Using deep learning for image-based plant disease detection. Frontiers in Plant Science. 2016 Sep 22;7(September). 1419. https://doi.org/10.3389/fpls.2016.01419.

[7] Ferentinos, Konstantinos. Deep learning models for plant disease detection and diagnosis. Computers and Electronics in Agriculture. (2018). 145. 311-318. 10.1016/j.compag.2018.01.009.

[8] Erhan, Dumitru \& Bengio, Y. \& Courville, Aaron \& Vincent, Pascal. Visualizing Higher-Layer Features of a Deep Network. (2009) Technical Report, Univeristé de Montréal.

[9] C. Olah, A. Satyanarayan, I. Johnson, S. Carter, L. Schubert, K. Ye, A. Mordvintsev, Distill 3 (2018).

[10] M.D. Zeiler, R. Fergus, in: Lect. Notes Comput. Sci. (Including Subser. Lect. Notes Artif. Intell. Lect. Notes Bioinformatics), 2014.

[11] Leslie N. Smith. A disciplined approach to neural network hyper-parameters: Part 1 - learning rate, batch size, momentum, and weight decay. (2018) CoRR, abs/1803.09820.

[12] Howard J, Gugger S. Fastai: A Layered API for Deep Learning. Information. 2020; 11(2):108. https://doi.org/10.3390/info11020108.

[13] K. Simonyan, A. Vedaldi, A. Zisserman, in: 2nd Int. Conf. Learn. Represent. ICLR 2014 - Work. Track Proc., 2014.

[14] M. Sundararajan, A. Taly, Q. Yan, in: 34th Int. Conf. Mach. Learn. ICML 2017, 2017.

[15] J.T. Springenberg, A. Dosovitskiy, T. Brox, M. Riedmiller, in: 3rd Int. Conf. Learn. Represent. ICLR 
2015 - Work. Track Proc., 2015.

[16] R.R. Selvaraju, M. Cogswell, A. Das, R. Vedantam, D. Parikh, D. Batra, Int. J. Comput. Vis. 128 (2020).

[17] R.S. Olson, R.J. Urbanowicz, P.C. Andrews, N.A. Lavender, L.C. Kidd, J.H. Moore, in: Lect. Notes Comput. Sci. (Including Subser. Lect. Notes Artif. Intell. Lect. Notes Bioinformatics), 2016.

[18] Krotov, Dmitry, and John J. Hopfield. "Unsupervised learning by competing hidden units." Proceedings of the National Academy of Sciences 116.16 (2019): 7723-7731. Web. 20 June. 2021. 